\title{
CONHECIMENTO E USO DO GENGIBRE POR FAMILIARES DE ALUNOS DE DUAS unidades escolares de Alta Floresta/MT
}

\author{
Elisa dos Santos Cardoso ${ }^{1 *}$, Ana Aparecida Bandini Rossi ${ }^{1}$, Eliane Cristina Moreno de Pedri ${ }^{1}$, Rosimeire \\ Barboza Bispo², Uéliton Alves de Oliveira ${ }^{3}$, Vantuir Pereira da Silva ${ }^{4}$
}

\author{
${ }^{1}$ Programa de Pós-Graduação em Biodiversidade e Agroecossistemas Amazônicos, UNEMAT/Alta Floresta \\ ${ }^{2}$ Laboratório de Melhoramento Genético Vegetal, UENF/Campos dos Goytacazes. \\ ${ }^{3}$ Faculdade de Ciências Biológicas e Agrárias, Alta Floresta, MT, Brasil. ORCID: http://orcid.org/0000-0002-8617-6044. Participação: entrevistas, \\ análise de dados e redação. \\ ${ }^{4}$ Secretaria de Estado de Educação de Mato Grosso. \\ *E-mail para correspondência: elisabyo@gmail.com
}

Recebido em 29 de março de 2018. Aceito em 21 de dezembro de 2018. Publicado em 28 de dezembro de 2018.

\begin{abstract}
Resumo - O gengibre (Zingiber officinale Roscoe) é uma planta utilizada como condimento e fitoterápico. A utilização como fitoterápico segue o conhecimento tradicional, a sabedoria popular, um patrimônio cultural a ser preservado. Este estudo teve por objetivo realizar um levantamento quanto a utilização do gengibre como fitoterápico por familiares e alunos de duas unidades escolares de Alta Floresta, Mato Grosso. Informações socioeconômicas e relativas à utilização do gengibre foram obtidas por meio de entrevista estruturada e semiestruturada e observação participativa. Os resultados foram avaliados qualitativamente e quantitativamente. Os representantes das famílias entrevistadas são, em sua maioria, do sexo feminino, com idade entre 21 e 40 anos e todos declararam conhecer e utilizar o gengibre como fitoterápico, sendo que informações quanto as formas de uso são obtidas, em $90,2 \%$ dos casos, junto ao núcleo familiar. As maiores concordâncias de uso entre os entrevistados foram para o tratamento de gripe e infecção de garganta. Os resultados deste estudo reafirmam a necessidade de pesquisas científicas que registrem a utilização de plantas medicinais, preservando assim o patrimônio cultural e subsidiando pesquisas que visem o registro de fitoterápicos.
\end{abstract}

Palavras-chave: Fitoterápico, Zingiber officinale, patrimônio cultural.

\section{KNOWLEDGE AND USE OF GINGER BY RELATIVES OF STUDENTS FROM TWO SCHOOL UNITS IN ALTA FLORESTA/MT}

Aвstract - Ginger (Zingiber officinale Roscoe) is a plant used in herbal medicine and as a condiment. Traditional preparations of ginger are a form of cultural heritage which should be preserved. This study aimed to survey the use of ginger in phytotherapy by relatives and students of two school units of Alta Floresta, Mato Grosso state, Brazil. Socio-economic information related to the use of ginger was obtained through structured and semi-structured interviews and participative observation. Results were evaluated qualitatively and quantitatively. The majority of the representatives of the families interviewed were female, mostly between the ages of 21 and 40 years. All respondents affirmed their awareness of ginger and its phytotherapeutic properties; in $90.2 \%$ of cases knowledge of the medicinal properties of ginger was obtained from family members. Medicinal usages most frequently reported were treatment of colds and flu and treatment of throat infections. The results of this study reaffirm the importance of further surveys of the usage of medicinal plants, to preserve cultural heritage and also to direct future research of phytotherapy and the species used in herbal medicine.

Keywords: Phytotherapeutic, Zingiber officinale, CUltural heritage. 


\section{Conocimiento y uso del Jengibre por familiares de alumnos de dos unidades escolares de Alta Floresta/MT.}

Resumen - El jengibre (Zingiber officinale Roscoe) es una planta utilizada como condimento y fitoterápico. La utilización como fitoterápico sigue el conocimiento tradicional, la sabiduría popular, un patrimonio cultural a ser preservado. Este estudio tuvo por objetivo realizar un levantamiento en cuanto a la utilización del jengibre como fitoterápico por familiares y estudiantes de dos unidades escolares de Alta Floresta, MT. La información socioeconómica y relativa a la utilización del jengibre se obtuvo mediante una entrevista estructurada y semiestructurada y una observación participativa. Los resultados fueron evaluados cualitativamente y cuantitativamente. Los representantes de las familias entrevistadas son, en su mayoría, del sexo femenino, con edad entre 21 y 40 años y todos declararon conocer y utilizar el jengibre como fitoterápico, siendo que informaciones como las formas de uso se obtienen, en el 90,2\% en los casos, junto al núcleo familiar. Las mayores concordancias de uso entre los entrevistados fueron para el tratamiento de la gripe e infección de garganta. Los resultados de este estudio reafirman la necesidad de investigaciones científicas que registren la utilización de plantas medicinales, preservando así el patrimonio cultural y subsidiando investigaciones que visen el registro de fitoterápicos.

Palabras clave: Fitoterápico, Zingiber officinale, patrimonio cultural.

\section{INTRODUÇÃO}

A utilização de recursos vegetais para prevenção, tratamento ou cura de doenças é uma prática milenar, sendo que os primeiros registros datam de 4000 a.C., enquanto o primeiro registro médico data de 2100 a.C. (Firmo et al. 2011), constituindo-se assim na forma mais antiga de práticas medicinais.

Plantas utilizadas pela medicina tradicional representam um recurso biológico utilizado na produção de remédios caseiros e como matéria prima pela indústria farmacêutica. Nas últimas décadas, a medicina tradicional, considerada como popular e informal, foi classificada como alternativa ou complementar, quando os tratamentos substituem ou são utilizados juntamente com os indicados pela medicina convencional (Spadacio e Barros 2009).

Terapias tradicionais por meio de recursos vegetais estão associadas aos saberes populares, sendo estes construídos ao longo da história e transmitidos ao longo das gerações. Esses saberes constituem, portanto, um patrimônio cultural que deve ser preservado, haja vista que compreendem um vasto acervo de conhecimento sobre plantas utilizadas pela medicina tradicional, suas indicações e formas de preparo (Almeida 2011; Sales et al. 2015).

Registros científicos da utilização de plantas como fitoterápicos são importantes não só por preservarem os saberes populares, mas também por fornecerem informações úteis a pesquisas na área farmacêutica. Algumas plantas medicinais como, por exemplo, o ginseng (Panax spp.) e o gengibre (Zingiber officinale Roscoe) são utilizadas pela medicina tradicional e exploradas pela indústria farmacêutica e o registro de sua utilização data de 1500 a.C., no manuscrito Egípicio "Ebers Papirus" e no texto sagrado "Ayurveda", respectivamente (Firmo et al. 2011).

Algumas propriedades terapêuticas do gengibre já foram comprovadas cientificamente (Sousa et al. 2013), e atualmente, a espécie consta da Relação Nacional de Plantas Medicinais de Interesse ao Sistema Único de Saúde (RENISUS), cujo objetivo é orientar estudos e pesquisas que possam subsidiar a utilização de fitoterápicos de forma segura e eficaz, tornando-se mais uma ferramenta a ser utilizada em estratégias para melhoria na qualidade da saúde pública no Brasil (Brasil 2009).

Dentre as enfermidades tratadas com fitoterápicos tradicionais preparados com o gengibre estão a gripe e problemas gastrointestinais (Grandi 2014), sendo que estudos demonstram que a espécie também atua como agente gastroprotetor (Haniadka et al. 2013), anti-inflamatório, diurético, expectorante, antioxidante, antimicrobiano e hepatoprotetora, além de contribuir para diminuição e controle de glicemia (Kubra e Rao 2012; Palatty et al. 2013). Pesquisas recentes indicam 
que o potencial antiproliferativo e antiangiogênico do gengibre apresenta a espécie como promissora para produção de medicamentos para tratamento de câncer (Karna et al. 2012; Fuzzer et al. 2017).

O gengibre é uma monocotiledônea herbácea, perene, com rizoma revestido por epiderme de cor pardacenta, raízes adventícias, pseudocaules eretos e pedúnculos com flores hermafroditas (Elpo e Negrelle 2004). É originário da Ásia e cultivado em grande escala apenas pelos estados do Espírito Santo, São Paulo, Paraná e Santa Catarina (Sousa et al. 2013). Todavia, o gengibre é cultivado por pequenos produtores e mantenedores de quintais, urbanos ou rurais, para consumo próprio ou comercialização em comércios ou feiras locais, sendo utilizado tanto para fins medicinais quanto culinários.

Diante do exposto, este estudo teve como objetivo realizar um levantamento quanto ao conhecimento tradicional e utilização de gengibre pela comunidade escolar de duas unidades escolares do município de Alta Floresta, MT, e assim contribuir para preservação do patrimônio cultural quanto a utilização culinária e medicinal da espécie, bem como servir de subsídio para pesquisas que visem o registro de produtos fitoterápicos.

\section{Material e Métodos}

Area de estudo

A pesquisa foi realizada com familiares e alunos das Escolas Estaduais Jardim Universitário (EE J.U.) e Rui Barbosa (EE R.B.), localizadas, respectivamente, nos bairros Jardim Universitário e Cidade Alta, em Alta Floresta, norte de Mato Grosso (Figura 1). O município está localizado a $797 \mathrm{~km}$ da capital do estado, Cuiabá e possui, aproximadamente, 50.189 habitantes (IBGE, 2017). O clima do município é do tipo Am (tropical e subtropical), com precipitação pluviométrica anual entre 2500 e 2100 milímetros e temperaturas médias superiores a $26{ }^{\circ} \mathrm{C}$ (Alvares et al., 2013).

Figura 1. Localização geográfica das comunidades escolares participantes da pesquisa, Alta Floresta, Mato Grosso, Brasil.
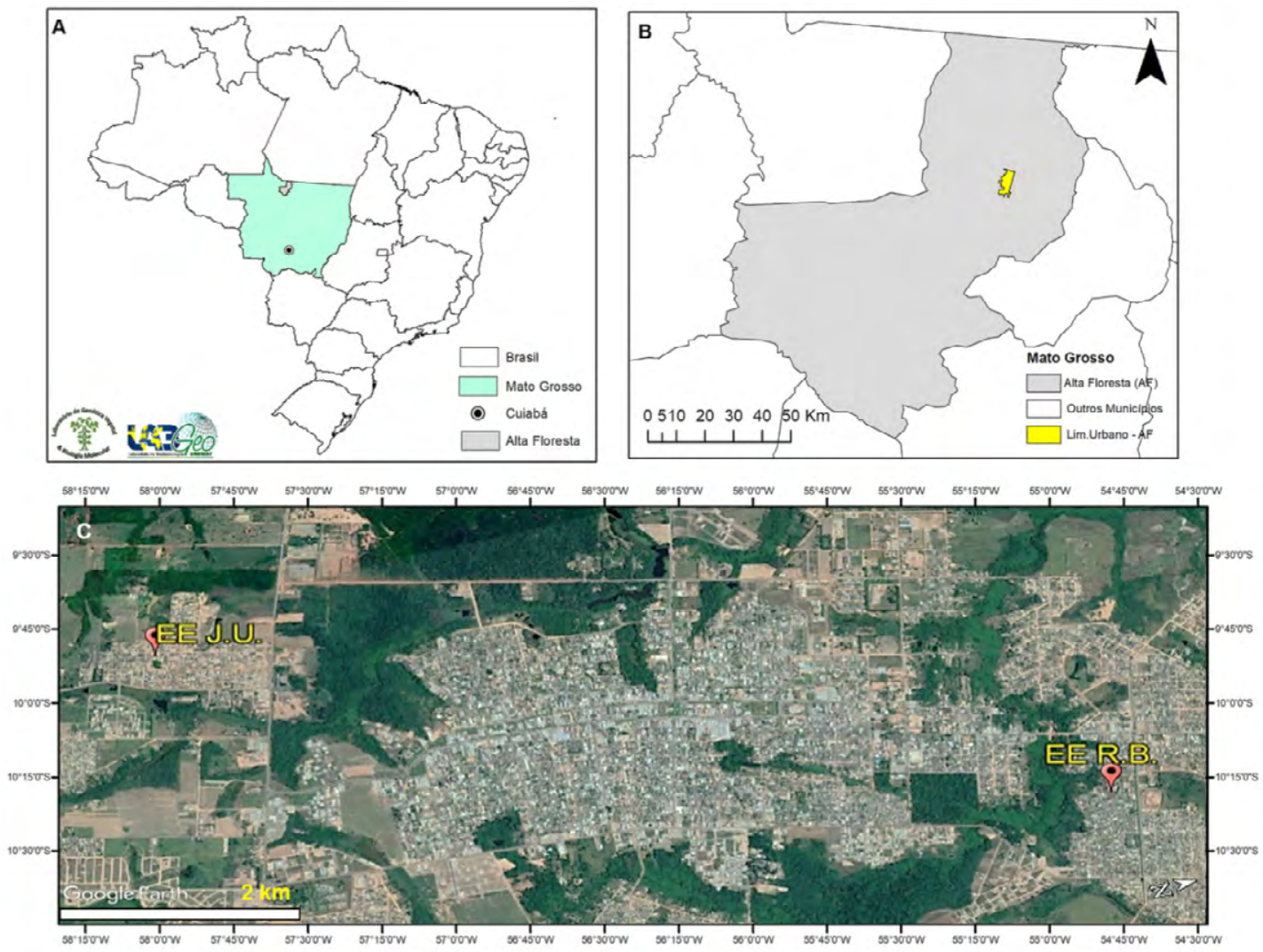


\section{Coleta E ANÁlise dos DADOS}

O estudo foi realizado entre os meses de agosto de 2017 e janeiro de 2018 a partir de levantamento realizado com estudantes de Ensino Fundamental e Médio das Escolas Estaduais Jardim Universitário (EE J.U.) e Rui Barbosa (EE R.B.) do município de Alta Floresta, MT, para identificação das famílias que conhecem e/ou utilizam o gengibre para fins fitoterápicos.

O projeto de pesquisa foi submetido à apreciação do Comitê de Ética em Pesquisa da Universidade do Estado de Mato Grosso (UNEMAT) sob o número 52641915.8.0000.5166, parecer consubstanciado 1.507.091 aprovado em 19 de dezembro de 2016.

A primeira etapa da pesquisa consistiu em um levantamento, junto a EE J.U e a EE R.B., quanto ao uso do gengibre como planta medicinal. No primeiro contato com os alunos, foram apresentados o projeto de pesquisa foi apresentado e o Termo de Consentimento Livre e Esclarecido (TCLE). Em se tratando de alunos menores de idade, o TCLE foi encaminhado aos responsáveis para que autorizassem sua participação na pesquisa. Após a assinatura deste TCLE pelos responsáveis, os alunos menores de idade receberam um Termo de Assentimento Livre e Esclarecido (TALE). Ao receber o TCLE ou o TALE, os alunos ou responsáveis eram livres para concordar, ou não, em participar da pesquisa. Após a assinatura dos termos, os alunos responderam à um questionário onde, além das informações pessoais, foram questionados sobre o conhecimento e utilização do gengibre como planta medicinal.

$\mathrm{Na}$ segunda etapa da pesquisa foram realizadas visitas às famílias de alunos que informaram utilizar o gengibre como planta medicinal. Nesta visita, o projeto foi explicado novamente e o TCLE apresentado. Assim como na primeira etapa, havia a liberdade em escolher participar ou não da pesquisa. Após a assinatura do TCLE, a entrevista com questões estruturada e semiestruturada foi realizada, bem como com a observação participativa (Minayo 2014).

O guia de entrevista foi divido em duas partes: a primeira, contendo 11 questões, relativas a informações pessoais e socioeconômicas e a segunda, composta por 12 questões, relacionadas ao conhecimento e utilização do gengibre. As respostas dos entrevistados foram registradas e analisadas quantitativamente para avaliar a importância relativa do uso pelos entrevistados.

Os Índices de Concordância de Uso foram calculados de acordo com Amorozo e Gély (1988), com adequações. A porcentagem de concordância para cada forma de utilização (CUP), revelou a importância relativa do gengibre como fitoterápico. Para a variável CUP, foram considerados as formas de utilização citadas por 3 ou mais entrevistados. Para obtenção do NECUP (número de entrevistados que citam determinada forma de uso) foram contabilizadas todas as formas de utilização. Os cálculos foram realizados de acordo com a equação 1:

$$
\mathrm{CUP}=\mathrm{NECUP} / \mathrm{NECC} \times 100
$$

onde,

CUP $=\%$ de concordância para cada utilização

NECUP $=n^{\circ}$ de entrevistados que citam determinada utilização para o gengibre

NECC $=n^{\circ}$ de entrevistados que citam a categoria de utilização

Posteriormente, foram calculados os fatores de correção para cada uma das categorias (equação 2) e o valor de importância de cada forma de utilização relativo à categoria mais citada pelos entrevistados (equação 3).

sendo,

$$
\begin{gathered}
\mathrm{FC}=\mathrm{NECC} / \mathrm{NECCM} \\
\mathrm{CUPc}=\mathrm{CUP} \times \mathrm{FC}
\end{gathered}
$$

$\mathrm{FC}=$ fator de correção da categoria

NECC $=n^{\circ}$ de entrevistados que citam a categoria de utilização

$\mathrm{NECCM}=\mathrm{n}^{\circ}$ de entrevistados que citaram a categoria mais utilizada

CUPc $=$ valor de importância relativo à forma de utilização mais citada pelos entrevistados 


\section{Resultados e Discussão}

$\mathrm{Na}$ primeira etapa da pesquisa foi realizado um levantamento com 138 alunos, sendo 67 do Ensino Médio da Modalidade Regular, 28 e 42 do Ensino Fundamental e Médio, respectivamente, da Modalidade de Educação de Jovens e Adultos (EJA). Dentre estes alunos, 58,70\% tem idade igual ou inferior a 20 anos, 78,26\% cursam o Ensino Médio, 58,70\% são solteiros e 57,25\% são do sexo feminino (Figura 2A).

Quando questionados quanto ao conhecimento sobre o gengibre 94,20\% declaram conhecê-la e 70,29\% informaram que a família a utiliza como fitoterápico, embora apenas $21,81 \%$ a cultivem em sua propriedade (Figura 2B).

Figura 2. (A) Distribuição dos alunos entrevistados, segundo o sexo, a idade, a escolaridade (Esc.) e o Estado Civil. F = Feminino; $\mathbf{M}=$ Masculino; EM = Ensino Médio; EF = Ensino Fundamental; $\mathbf{S T}=$ Solteiro; $\mathbf{C S}=$ Casado; AS = Amasiado; UE = União Estável; NM = Namorando; DV = Divorciado $;$ NR = Não Responderam. (B) Classificação das respostas quanto ao conhecimento sobre o gengibre.
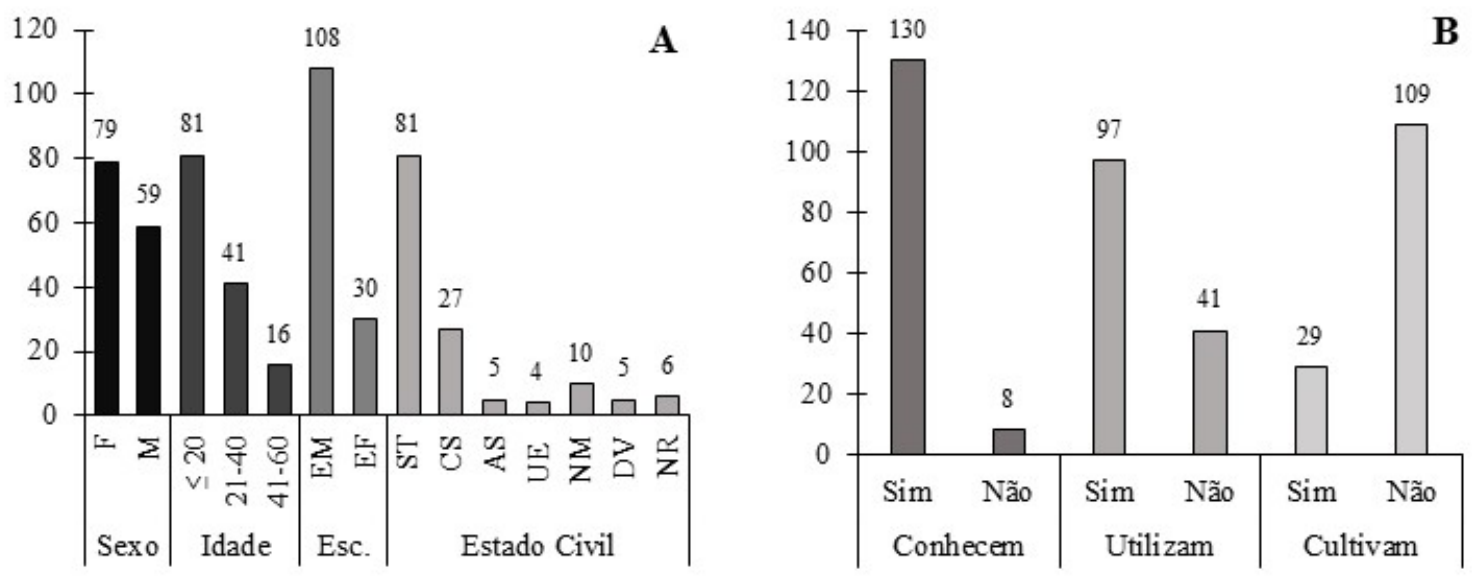

Dentre os entrevistados que informaram utilizar o gengibre como fitoterápico, foram escolhidos, aleatoriamente, 60 participantes, dos quais as famílias foram visitadas para realização da segunda etapa da pesquisa, sendo que cinco (5) famílias optaram por não participar e quatro (4) não foram encontradas.

Os entrevistados na segunda etapa da pesquisa são, na sua maioria, do sexo feminino (80,39\%), casados $(49,02 \%)$, tem entre 21 e 40 anos de idade $(50,98 \%)$ e são naturais, principalmente, dos estados de Mato Grosso $(33,33 \%)$ e Paraná $(27,45 \%)$ (Figuras 3A e 3B).

Figura 3. Distribuição dos entrevistados de acordo com: $(\mathrm{A})$ sexo, estado civil $\mathrm{e}$ idade. $(\mathrm{B})$ naturalidade. $\mathrm{F}=$ Feminino; $\mathrm{M}=$ Masculino; $\mathrm{ST}=$ Solteiro; $\mathrm{CS}$ = Casado; VV = Viúvo; UE/AS = União Estável/Amasiado; $\mathbf{M T}=$ Mato Grosso; PR = Paraná; MS = Mato Grosso do Sul; GO = Goiás; ES = Espirito Santo; MG = Minas Gerais;

RS = Rio Grande do Sul; PA = Pará; AL = Alagoas.
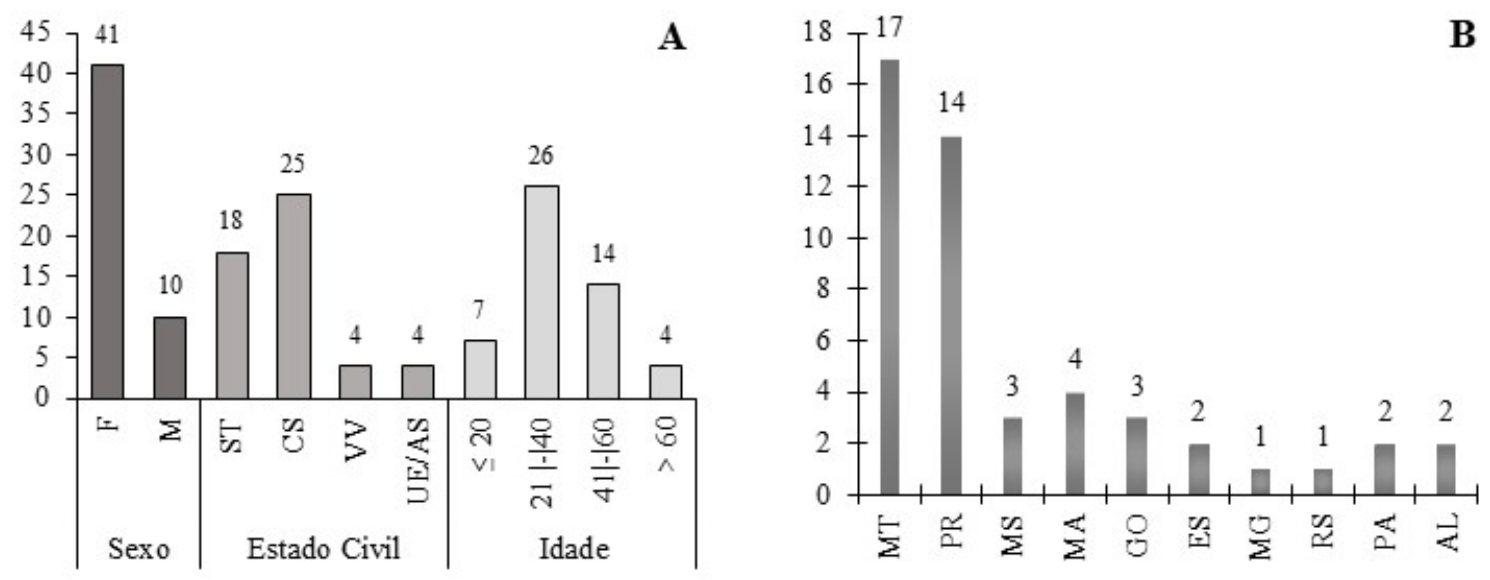
A naturalidade dos entrevistados está relacionada ao processo de colonização do município e à idade dos mesmos, haja vista que pequenos agricultores e/ou comerciantes da região Sul do país se deslocaram para o norte de Mato Grosso em busca de melhor qualidade de vida e seus filhos aqui permaneceram e constituíram família (Seluchinesk, 2008).

Os entrevistados residem na zona urbana do município de Alta Floresta e se encontram distribuídas em 15 bairros distintos, sendo que 33,33\% residem no mesmo bairro há, no máximo, cinco anos, (Figura 4A e 4B). O número de moradores por residência variou entre um (1) e onze (11), sendo que em 35,29\% eram apenas três moradores.

Figura 4. Distribuição dos entrevistados quanto ao: (A) bairro do município de Alta Floresta em que residem e (B) tempo de residência no mesmo. J.U. = Jardim Universitário; M.L. = Mirante dos Lagos; J.O. = Jardim das Oliveiras; J.P. = Jardim das Primaveras; J.R. = Jardim Renascer; J.T.: Jardim Tropical; P.O. Parque dos Oitis; B.E. $=$ Boa Esperança; J.F. = Jardim das Flores; G. = Guaraná; V.N.=Vila Nova; B.P. $=$ Bom Pastor; C.A. $=$ Cidade Alta; C.B. = Cidade Bela; B.J. = Bom Jardim.
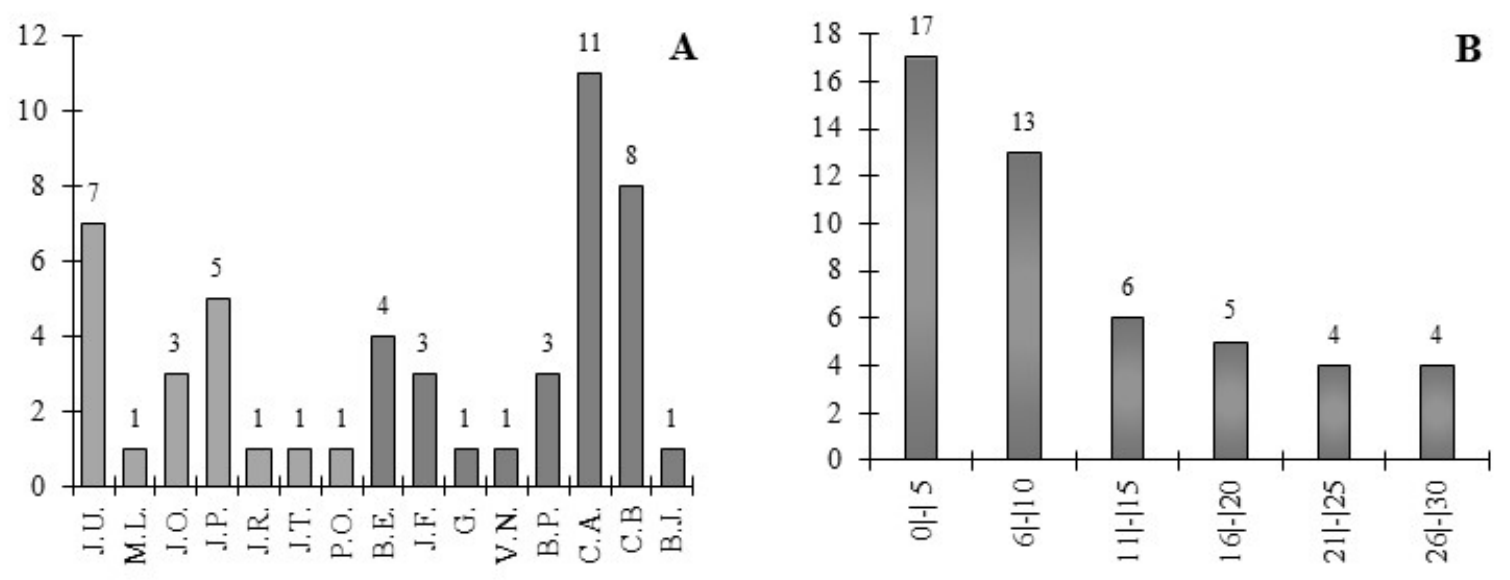

Quanto à escolaridade, 29,41\% dos entrevistados possuem apenas o Ensino Fundamental Incompleto, dois (2) se declararam analfabetos e apenas três (3) possuem o Ensino Superior Completo. Foram citadas 19 ocupações/ fontes de renda distintas, dentre as quais se destacam "do lar" (39,21\%) e autônomo (14,26\%). A renda das famílias entrevistadas é, em sua maioria, de até três (3) salários mínimos (92,16\%), sendo que do total de famílias, 11 são beneficiárias de algum programa de assistência social.

Todos os entrevistados declararam conhecer o gengibre e utilizar apenas o rizoma como fitoterápico, dos quais 38 declararam utilizar também como ingrediente culinário (Tabela 1). Apenas 20 entrevistados possuem a planta em sua propriedade e declararam que não existem recomendações especiais para o plantio, exceto que o mesmo deve ser feito em terra fofa.

Entrevistado 01: "Cultivar em local de terra fofa."

Entrevistado 02: “(...) planta agora, por esse tempo, uma terra bem adubada. Planta, rega, cuida e, quando for lá pro mês de junho e julho, colhe-se as raízes."

Quanto aos que não possuem, 74,19\% adquirem o rizoma do gengibre no comércio local (mercado ou feira) e os demais, com familiares, vizinhos e amigos. 
Tabela 1. Categorias de uso e formas de utilização do gengibre no município de Alta Floresta. N.C. = número de citações

\begin{tabular}{llcl}
\hline Categoria & Formas de Utilização & N.C & Preparo \\
\hline \multirow{5}{*}{ Culinária } & Tempero & 22 & Ralado; Em pó \\
\cline { 2 - 4 } & Quentão & 7 & Decocção \\
\cline { 2 - 4 } & Bala & 5 & Decocção \\
\cline { 2 - 4 } & Bolachas & 1 & Ralado \\
\cline { 2 - 4 } & Bolos & 1 & Ralado \\
\cline { 2 - 4 } & Licor & 1 & Decocção \\
\cline { 2 - 4 } & Rapadura & 1 & Decocção \\
\hline \multirow{5}{*}{ Medicinal } & Infecção de Garganta & 26 & Infusão; Decocção; In natura \\
\cline { 2 - 4 } & Gripe & 27 & Infusão; Decocção; Xarope \\
\cline { 2 - 4 } & Inflamações & 4 & Infusão; Maceração \\
\cline { 2 - 4 } & Problemas digestivos & 3 & Infusão; In natura \\
\cline { 2 - 4 } & Emagrecer & 13 & In natura; Infusão \\
\cline { 2 - 4 } & Câncer & 3 & In natura \\
\cline { 2 - 4 } & Tosse & 3 & Infusão; Decocção \\
\cline { 2 - 4 } & Resfriado & 12 & Infusão; Decocção \\
\cline { 2 - 4 } & Queda de cabelo & 1 & Azeite \\
\cline { 2 - 4 } & Diabetes & 1 & Decocção \\
\cline { 2 - 4 } & Cólica & 1 & Decocção \\
\cline { 2 - 4 } & Virose & Decocção \\
\hline
\end{tabular}

As formas de utilização do gengibre com maior concordância (CUPc) entre os entrevistados estão apresentadas na tabela 2, destacando-se a gripe e a dor de garganta (53\% e 51\%, respectivamente) na categoria medicinal e o tempero (43\%), na categoria culinária.

As principais aplicações do gengibre como fitoterápico citadas neste estudo foram para gripe e infecções de garganta, sendo, portanto, concordante com as indicações de utilização do gengibre presentes no estudo etnofarmacológico realizado nos biomas da Amazônia e Mata Atlântica (Di Stasi e Hiruma-Lima 2002), no levantamento do uso de plantas medicinais por moradores de Governador Valadares, MG (Brasileiro et al. 2008), de Demerval Lobão, PI (Aguiar e Barros 2012), de Lima Duarte, MG (Oliveira e Menini Neto 2012) e no distrito de Marudá, PA (Flor e Barbosa 2015). 
Tabela 2. Principais formas de utilização e concordância quanto a utilização de gengibre no município de Alta Floresta, Mato Grosso. NECC $=\mathbf{n}^{\circ}$ de entrevistados que citaram a categoria de utilização; $\mathbf{N E C U P}=\mathrm{n}^{\circ}$ de entrevistados que citaram determinada forma de utilização; CUP = índice de concordância de uso principal; FC = fator de correção; CUPc = CUP corrigido.

\begin{tabular}{|c|c|c|c|c|c|c|}
\hline Categoria & Formas de utilização & NECC & NECUP & CUP & FC & CUPc \\
\hline \multirow{8}{*}{ Medicinal } & Infecção de Garganta & 51 & 26 & $51 \%$ & 1,00 & $51 \%$ \\
\hline & Gripe & 51 & 27 & $53 \%$ & 1,00 & $53 \%$ \\
\hline & Inflamações & 51 & 4 & $8 \%$ & 1,00 & $8 \%$ \\
\hline & Problemas digestivos & 51 & 3 & $6 \%$ & 1,00 & $6 \%$ \\
\hline & Emagrecer & 51 & 13 & $25 \%$ & 1,00 & $25 \%$ \\
\hline & Câncer & 51 & 3 & $6 \%$ & 1,00 & $6 \%$ \\
\hline & Tosse & 51 & 3 & $6 \%$ & 1,00 & $6 \%$ \\
\hline & Resfriado & 51 & 12 & $24 \%$ & 1,00 & $24 \%$ \\
\hline \multirow{3}{*}{ Culinária } & Tempero & 38 & 22 & $58 \%$ & 0,75 & $43 \%$ \\
\hline & Quentão & 38 & 7 & $18 \%$ & 0,75 & $14 \%$ \\
\hline & Bala & 38 & 5 & $13 \%$ & 0,75 & $10 \%$ \\
\hline
\end{tabular}

Marmitt et al. (2015), ao realizarem levantamento sistemático em três bancos de dados quanto a pesquisas relativas às plantas da Relação Nacional de Plantas Medicinais de Interesse ao Sistema Único de Saúde (RENISUS) no tratamento de infecções/inflamações, apontam o gengibre como a segunda planta mais pesquisada para esta finalidade, ficando atrás apenas do número de estudos sobre o açafrão (Curcuma longa L.), que também pertence à família Zingiberaceae.

A principal fonte de informação sobre as propriedades medicinais do gengibre citada pelos entrevistados foi a família $(90,20 \%)$, sendo estas as responsáveis por transmitir não apenas informações quanto as indicações como também quanto a forma de preparo dos fitoterápicos. A importância da família na manutenção do patrimônio cultural relacionado as plantas medicinais também foi relatada por Paulino et al. (2011) ao realizar levantamento quanto ao conhecimento sobre plantas medicinais entre alunos da Universidade Federal do Semiárido, em Mossoró, Rio Grande do Norte, e por Oliveira et al. (2016) ao realizarem pesquisa junto a alunos de Ensino Fundamental de duas escolas de Viçosa do Ceará.

Além da decocção e infusão, que são as formais mais comuns de preparação do gengibre como fitoterápico, foram descritas também a preparação de xarope e diferentes formas de consumo in natura. Um dos entrevistados relatou utilizar o azeite de gengibre para combater a queda de cabelo:

Entrevistado 35: “maceta o gengibre e frita no óleo de oliva. Quando tostar, desligar o fogo e filtrar o óleo para passar no cabelo."

\section{ConClusões}

A principal categoria de utilização do gengibre pelos entrevistados é a medicinal, embora muitos também o utilizem na culinária, especialmente como tempero. O conhecimento dos entrevistados neste estudo quanto a forma de utilização e modo de preparo do Z. officinale como fitoterápico é transmitido ao longo das gerações, o que reafirma a importância de pesquisas científicas que registram esse patrimônio cultural, e assim, além de preservá-lo, contribuam para estudos e pesquisas com intuito de promover o registro de produtos fitoterápicos. 


\section{Agradecimentos}

À Secretaria de Educação do Estado de Mato Grosso (SEDUC/MT), à Coordenação de Aperfeiçoamento de Pessoal de Nível Superior (CAPES), ao Conselho Nacional de Desenvolvimento Científico e Tecnológico (CNPq), à Universidade do Estado de Mato Grosso (UNEMAT) e ao Programa de Pós-Graduação em Biodiversidade e Agroecossistemas Amazônicos, UNEMAT.

\section{REFERÊNCIAS}

Aguiar LCGG, Barros RFM. 2012. Plantas medicinais cultivadas em quintais de comunidades rurais no domínio do cerrado piauiense (Município de Demerval Lobão, Piauí, Brasil). Revista Brasileira de Plantas Medicinais, 14(3): 419-432.

Almeida MZ. 2011. Plantas medicinais. 3.ed. Salvador: EDUFBA, 221p.

Alvares CA, Stape JL, Sentelhas PC, Gonçalves JLM, Sparovek G. 2013. Koppen's climate classification map for Brazil. Meteorologische Zeitschrift, 22(6):711-728. DOI: 10.1127/0941-2948/2013/0507.

Amorozo MCM, Gély A. 1988. Uso de plantas medicinais por caboclos do Baixo Amazonas. Barcarena, PA, Brasil. Boletim do Museu Emílio Goeldi, Série Botânica, Sup., 4:47-129.

Brasil. Ministério da Saúde. Porta da Saúde. Plantas Medicinais de Interesse ao SUS: RENISUS. Disponível em: < http:// portalarquivos2.saude.gov.br/images/pdf/2017/junho/06/renisus.pdf >. Acesso em 8 jan 2018.

Brasil. Resolução da Diretoria Colegiada n.26, 13 de maio de 2014. O registro de medicamentos fitoterápicos e o registro e a notificação de produtos tradicionais fitoterápicos. Brasilia, DF, mai 2014.

Brasileiro BG, Pizziolo VR, Matos DS, Germano AM, Jamal CM. 2008. Plantas medicinais utilizadas pela população atendida no "Programa de Saúde da Família", Governador Valadares, MG, Brasil. Revista Brasileira de Ciências Farmacêuticas, 44(4):629-633.

Bruning MCR, Mosegui GBG, Vianna CMM. 2012. A utilização da fitoterapia e de plantas medicinais em unidades básicas de saúde nos municípios de Cascavel e Foz do Iguaçu - Paraná: a visão dos profissionais de saúde. Ciências \& Saúde Coletiva, 17(10):2675-2685. DOI: 10.1590/S1413-81232012001000017.

Di Stasi LC, Hiruma-Lima CA. 2002. Plantas Medicinais na Amazônia e na Mata Atlântica. São Paulo: UNESPE, 600p.

Elpo ERS, Negrelle RRB. 2004. Zingiber officinale Roscoe: aspectos botânicos e ecológicos. Visão Acadêmica, 5(1):27-32.

Fernandes TM. 2004. Plantas Medicinais: memória da ciência no Brasil. Rio de Janeiro: Fiocruz, 260p.

Firmo WCA, Menezes VJM, Passos CEC, Dias CN, Alves LPL, Dias ICL, Santos Neto M, Olea RSG. 2011. Contexto histórico, uso popular e concepção científica sobre plantas medicinais. Cadernos de Pesquisas, 18(esp.):90-95.

Flor ASSO, Barbosa WLR. 2015. Sabedoria popular no uso de plantas medicinais pelos moradores do Bairro do Sossego no Distrito de Marudá-PA. Revista Brasileira de Plantas Medicinais, 17(4):757-768.

Fuzer AM, Lee S, Mott, JD, Cominetti MR. 2017. [10] - gingerol reverts malignant phenotype of breast cancer cells in 3D culture. Journal of Cellular Biochemistry, 118(9):2693-2699. DOI: 10.1002/jcb.25906. 
Grandi TSM. 2014. Tratado de plantas medicinais: mineiras, nativas e cultivadas. Belo Horizonte: Adaequatio Estúdio, $1204 \mathrm{p}$.

Haniadka R, Saldanha E, Sunita V, Palatty PL, Fayad R, Baliga MS. 2013. A review of the gastroprotective effects of ginger (Zingiber officinale Roscoe). Food \& Function, 4(6):845-855. DOI:10.1039/C3FO30337C.

IBGE. Instituto Brasileiro de Geografia e Estatística. Panorama - Alta Floresta, IBGE 2017. Disponível em: < https:// cidades.ibge.gov.br/brasil/mt/alta-floresta/panorama>. Acesso em 7 jan 2018.

Karna P, Chagani S, Gundala SR, Rida PCG, Asif G, Sharma V, Gupta, MV, Aneja R. 2012. Benefits of whole ginger extract in prostate cancer. British Journal of Nutrition, 107(4):473-484. DOI: 10.1017/S0007114511003308.

Kubra R, Rao JM. (2012) An Impression on Current Developments in the Technology Chemistry, and Biological Activities of Ginger (Zingiber officinale Roscoe). Critical Reviews in Food Science and Nutrition, 52(8):651-688. DOI: 10.1080/10408398.2010.505689.

Marmitt DJ, Rempel C, Goettert MI, Silva AC. 2015. Plantas medicinais da RENISUS com potencial anti-inflamatório: Revisão sistemática em três bases de dados científicas. Fitos, 9(2):73-150. DOI: 10.5935/2446-4775.20150011.

Minayo, M.C.S. 2014. O desafio do conhecimento: pesquisa qualitativa em saúde. 13.ed. São Paulo: Hucitec, 407p.

Oliveira ER, Minini Neto L. 2012. Levantamento de plantas medicinais utilizadas pelos moradores do povoado de Manejo, Lima Duarte - MG. Revista Brasileira de Plantas Medicinais, 14(2):311-320.

Oliveira IP, Araújo MP, Meireles VJS, Lemos, JR. 2016. Conhecimento de plantas medicinais e relação com o ambiente por alunos de duas escolas de ensino fundamental do município de Viçosa do Ceará, Ceará. Pesquisa em Educação Ambiental, 11(1):81-93. DOI: 10.18675/2177-580X.vol11.n1.p.81-93.

Palatty PL, Haniadka R, Valder B, Arora R, Baliga MS. 2013. Ginger in the prevention of nausea and vomiting: a review. Critical Reviews in Food Science and Nutrition, 53(7):659-669. DOI: 10.1080/10408398.2011.553751.

Paulino RC, Henriques GPSA, Coelho MFB, Maia SSSS. 2011. Conhecimento sobre plantas medicinais entre alunos da Universidade Federal do Semiárido, Mossoró, RN. Revista Verde de Agroecologia e Desenvolvimento Sustentável, 6(4):78-90.

Sales MDC, Sartor EB, Gentilli RML. 2015. Etnobotânica e etnofarmacologia: medicina tradicional e bioprospecção de fitoterápicos. Revista SALUS, 1(1):17-26. DOI: 10.5935/2447-7826.20150003.

Seluchinesk RDC. De heróis a vilões: imagem e autoimagem dos colonos da Amazônia mato-grossense. 2008. 263f. Tese (Doutorado em Desenvolvimento Sustentável) - Centro de Desenvolvimento Sustentável da Universidade de Brasília. Brasília, 2008.

Sousa LS, Silva ÍRC, Assis DJ, Pascoal DRC, Druzian JI. 2013. Estudo prospectivo sobre as propriedades terapêuticas do Zingiber officinale (gengibre) com ênfase na ação antimicrobiana. GEINTEC, 3(5):427-436. DOI: 10.7198/geintec.v3i5.332

Spadacio C, Barros NF. 2009. Terapêuticas convencionais e não convencionais no tratamento do câncer: os sentidos das práticas religiosas. Interface, 13(30):45-52. DOI: 10.1590/S1414-32832009000300005. 